\title{
MicroRNA-588 suppresses tumor cell migration and invasion by targeting GRN in lung squamous cell carcinoma
}

\author{
LI QIAN ${ }^{1}$, LONGLONG LIN $^{2}$, YUFENG DU ${ }^{1}$, XIAOYAN HAO ${ }^{1}$, YUZE ZHAO ${ }^{1}$ and XUEJUN LIU ${ }^{1}$ \\ ${ }^{1}$ Department of Geriatrics, First Hospital of Shanxi Medical University, Taiyuan, Shanxi 030001; \\ ${ }^{2}$ Renji Hospital, Shanghai Jiao Tong University School of Medicine, Shanghai 200032, P.R. China
}

Received August 4, 2015; Accepted August 3, 2016

DOI: $10.3892 / \mathrm{mmr} .2016 .5643$

\begin{abstract}
MicroRNAs (miRNAs) have been demonstrated to be critical in regulating tumor development and progression. The present study investigated the expression of miR-588 using reverse transcription-quantitative polymerase chain reaction analysis in 85 cases of lung squamous cell carcinoma (SCC), and observed the correlation between the expression of miR-588 with clinical pathologic features. The results indicated that the expression of miR-588 was predominantly lower in the tumor samples, compared with non-tumorous samples, and was negatively associated with tumor stages and lymph node invasion. The present study also examined the significance of the expression of miR-588 in SCC using gain- and loss-of-function analyses. It was found that miR-588 inhibited tumor cell migration and invasion. In addition, it was revealed that the overexpression of miR-588 in SCC cells reduced the mRNA and protein levels of progranulin (GRN), whereas miR-588 silencing increased the expression of GRN. A luciferase activity assay showed that miR-588 was able to directly bind to the 3 'untranslated region of GRN and regulate its expression. Furthermore, it was found that the expression of GRN was inversely correlated with the expression of miR-588 in 85 paired SCC samples. These results indicated that GRN was involved in the miR-588-mediated suppressive functions in the progression of SCC.
\end{abstract}

\section{Introduction}

Lung cancer is a major contributor to mortality rates worldwide, with a mortality rate of $>160,000$ in America and a 5 -year survival rate of $\sim 16 \%$ (1). Lung squamous cell carcinoma (SCC) is defined as a common type of non-small cell lung cancer (NSCLC), which accounts for $\sim 80 \%$ of all cases

Correspondence to: Professor Xuejun Liu, Department of Geriatrics, First Hospital of Shanxi Medical University, 85 Jiefang South Road, Taiyuan, Shanxi 030001, P.R. China

E-mail: drlxj20041205@sina.com

Key words: microRNA-588, lung squamous cell carcinoma, invasion, progranulin, vascular endothelial growth factor of lung cancer (2,3). SCC is usually found in aged males and is correlated closely with smoking (4). SCC is also associated with high rates of metastasis and relapse (5). Therefore, further investigations on prognostic markers are required to manage the clinical treatments. Metastasis and angiogenesis are the hallmarks of cancer, and are the most important factors leading to cancer-associated mortality. Research has improved the understanding of metastasis (6), however, the precise molecular mechanisms remain to be fully elucidated. Although it has been revealed that mutations of several oncogenes, including Kirsten rat sarcoma viral oncogene homolog, anaplastic lymphoma kinase and epidermal growth factor receptor, or genome amplification contribute to the promotion and advanced stages of SCC $(7,8)$, further investigations to identify novel targets and elucidate molecular mechanisms are required.

MicroRNAs (miRNAs) are identified as a class of short non-coding RNAs, which regulate gene expression through binding to the 3'untranslated regions of the mRNAs of target genes, mediating its stability and degradation (9). In previous years, studies on miRNAs have shown that they are critical in cellular processes, including proliferation, apoptosis and motility. The dysregulation of miRNAs has been demonstrated in several types of tumor, including lung cancer, breast cancer and colon cancer, promoting tumorigenesis (10-12). Among these miRNAs, miR-588 has previously been reported in colorectal cancer-associated miRNAs (13), however, its role in lung cancer, particularly in SCC, has not been reported.

GRN (progranulin), also known as PC cell-derived growth factor, has been identified as a tissue and circulating biomarker for NSCLC (14). The deregulation of GRN has been found in several types of tumor, including breast cancer, prostate cancer, chronic lymphocytic leukemia and hepatocellular carcinoma (15). For example, in breast cancer, the expression of GRN has been found to be associated with the expression of estrogen receptor and the acquisition of resistance to drugs (16). Various studies have reported that the overexpression of GRN can activate multiple important pathways, including the mitogen-activated protein kinase, phosphoinositide 3-kinase/Akt and focal adhesion kinase pathways, promoting cell proliferation, invasion and tumorgenesis $(17,18)$. However, the significance and molecular mechanisms accounting for the upregulation of GRN remain to be fully elucidated. 
The aims of the present study were to provide evidence of the expression of miR-588 and its target gene, GRN, in SCC. The results showed that the expression of miR-588 was higher in non-tumor tissues, compared with SCC tissues, and that miR-588 suppressed SCC cell migration and invasion. In addition, GRN was identified as a potential target of miR-588, which was upregulated in SCC tissues, and the abnormal expression of GRN was involved in the tumor-suppressive functions mediated by miR-588.

\section{Materials and methods}

Human tissues and cell culture. A total of 85 pairs of human SCC and adjacent non-tumor tissues were collected between 2005 and 2014 at the Department of Pathology, First Hospital of Shanxi Medical University (Shanxi, China). The patients had no received systemic chemotherapy or radiotherapy prior to surgery. The clinical data collected consisted of age, gender, pathological tumor stage and lymph node invasion. The detailed information is shown in Table I. All specimens were histologically confirmed by two pathologists. Tumor subtype was examined based on the World Health Organization (WHO) 2004 criteria, and the staging was according to the TNM classification (UICC 2009) (19,20). All human tissues were acquired with informed consent and the approval of the Ethical Review Committee of the WHO of the Collaborating Center for Research in Human Production.

Human lung SCC cell lines (H226, H2170 and H1703) were purchased form American Type Culture Collection (ATCC; Manassas, VA, USA). All cell lines were maintained in RPMI 1640 medium (Gibco: Thermo Fisher Scientific, Inc., Waltham, MA, USA) supplemented with $10 \%$ fetal bovine serum (Hyclone: GE Healthcare Life Sciences, Logan, UT, USA) and antibiotics. The cells were incubated under 5\% $\mathrm{CO}_{2}$ and $37^{\circ} \mathrm{C}$ conditions according to ATCC.

Oligonucleotide transfection. The miR-588 mimics and small interfering (si)RNA against GRN (forward 5'-GCU UCCAAAGAUCAGGUAACATT-3' and reverse 5'-UGU UACCUGAUCUUUGGAAGCTT-3') were synthesized by GenePharma (Shanghai, China). The miR-588 inhibitors were synthesized by Thermo Fisher Scientific, Inc. Transfection was performed using Lipofectamine 2000 reagents according to the manufacturer's protocol.

Total RNA extraction and reverse transcription-quantitative polymerase chain reaction $(R T-q P C R)$ analysis. Total RNA was extracted from the 85 clinical tissue samples using TRIzol reagent (Invitrogen; Thermo Fisher Scientific, Inc,) according to the manufacturer protocol. Tissues were homogenized in the TRIzol reagent and then centrifuged at $13000 \times \mathrm{g}$ at $4^{\circ} \mathrm{C}$ for $20 \mathrm{~min}$. Reverse transcription was performed to convert the extracted RNA (200 ng) into cDNA with oligo (dT) primer $(25 \mathrm{pmol})$ and M-MLV reverse transcriptase (Takara Biotechnology Co., Ltd., Dalian, China) incubation at $42^{\circ} \mathrm{C}$ for $60 \mathrm{~min}$. RT-qPCR was performed using the SYBR-Green method (SYBR Premix Ex Taq II; Takara Biotechnology Co., Ltd.) with GRN-specific and $\beta$-actin primers (used at $0.4 \mu \mathrm{M}$ ) as an internal control. qPCR was performed using an ABI7500 real-time PCR system (Applied Biosystems: Thermo Fisher
Table I. Clinical characteristics of 85 patients with lung squamous cell carcinoma.

\begin{tabular}{lc}
\hline Characteristic & Cases (n) \\
\hline Age (years) & \\
$\quad<50$ & 26 \\
$\geq 50$ & 59 \\
Gender & \\
Male & 63 \\
Female & 22 \\
Stage & \\
I & 12 \\
II & 45 \\
III+IV & 28 \\
Lymph node invasion & \\
Negative & 55 \\
Positive & 30 \\
Smoking history & \\
Smoker & 48 \\
Non-smoker & 37 \\
\hline
\end{tabular}

Scientific, Inc.) with incubation at $95^{\circ} \mathrm{C}$ for $30 \mathrm{sec}$ (predenaturation) followed by 40 cycles of $95^{\circ} \mathrm{C}$ for $30 \mathrm{sec}$ and $60^{\circ} \mathrm{C}$ for $30 \mathrm{sec}$. The primers were as follows: GRN, forward 5'-ATC TTTACCGTCTCAGGGACTT-3' and reverse 5'-CCATCG ACCATAACACAGCAC-3' and $\beta$-actin, forward 5'-ATGATG ATATCGCCGCGCTC-3' and reverse 5'-CATCACGCCCTG GTGCC-3', respectively. The relative expression levels of GRN were calculated using the $\Delta \Delta \mathrm{Cq}$ method as described previously (21).

For measuring the expression of miR-588, TaqMan miRNA assays (Applied Biosystems: Thermo Fisher Scientific, Inc.) were performed according to the manufacturer's protocol, and U6 small nuclear RNA was used to normalize the relative gene expression.

Western blot analysis. The tissues or cells were lyzed in RIPA protein extraction reagent according to the manufacturer's protocol. The proteins were quantified using a BCA protein assay kit (Thermo Fisher Scientific, Inc.) according to the manufacturer's protocol. Subsequently, $50 \mu \mathrm{g}$ of total protein was loaded per lane of a $10 \%$ SDS gel, and then transferred onto a nitrocellulose membrane and blotted as described previously (22). The membrane was incubated with primary antibody at $4^{\circ} \mathrm{C}$ overnight. Following washing with phosphate-buffered saline, secondary antibody incubated with the membrane for $1 \mathrm{~h}$. Primary antibodies included anti-GRN (rabbit anti-human monoclonal antibody; 1:1,000 dilution; cat. no. ab108608; Abcam, Cambridge, UK), anti-vascular endothelial growth factor (VEGF; mouse anti-human monoclonal antibody; 1:1,000 dilution; cat. no. ab69479; Abcam) and anti-GAPDH (mouse anti-human monoclonal antibody; 1:2,000 dilution; cat. no. sc-365062; Santa Cruz Biotechnology, Inc., Santa Cruz, CA, USA). Secondary antibodies were goat horseradish peroxidase (HRP)-conjugated anti-mouse IgG 
(1:5,000; cat. no. sc-2005) and goat anti-rabbit $\operatorname{IgG}(1: 5,000$; cat. no. sc-2004) from Santa Cruz Biotechnology, Inc. The protein bands were visualized by enhanced chemiluminescent detection (Super Signal West Dura Extended Duration Chemiluminescent Substrate, Thermo Fisher Scientific, Inc.) and quantified by Quantity One software (version 4.4.036, Bio-Rad Laboratories, Inc., Hercules, CA, USA).

Immunohistochemical staining. All tissue specimens were surgically resected with adjacent normal lung tissues and fixed in $10 \%$ formalin followed by being embedded in paraffin. Serial $4-\mu \mathrm{m}$-thick sections were used for immunohistochemical analysis.

The formalin-fixed and paraffin-embedded sections were heated at $60^{\circ} \mathrm{C}$ for $30 \mathrm{~min}$ and dewaxed twice in xylene, followed by exposure to graded ethanol and finally water. Endogenous peroxidase was blocked by incubation in $0.3 \%$ hydrogen peroxide methanol for $10 \mathrm{~min}$, followed by antigen retrieval, which was performed via boiling in citrate buffer for $20 \mathrm{~min}$. The sections were incubated with a 1:200 dilution of anti-GRN primary antibody overnight at $4^{\circ} \mathrm{C}$. The following day, the sections were washed and then incubated with HRP-labeled anti-IgG for $30 \mathrm{~min}$. Finally, the sections were stained using diaminobenzidine substrate chromogen and counterstained with hematoxylin. All these steps were performed under a light microscope to control the reactions, and phosphate-buffered saline was performed instead of primary antibody as a negative control.

Evaluation of immunohistochemical staining. The immunostaining of GRN was quantified, blinded to the patients' clinical data, as described previously (23). In brief, the percentage of staining intensity was scored as follows: No staining $=0 ;<15 \%$ staining $=1$ (weak); $15-50 \%$ staining $=2$ (moderate) $;>50 \%$ staining $=3$ (strong). Furthermore, according to the percentage staining of the tumor cells and the sections, the scores were determined as intensity $\mathrm{x}$ percentage, to produce a final score of 0-300. The sections were distinguished into two groups, with either a high expression level of GRN or a low expression level of GRN.

Migration and invasion assays. For the migration assays, $2 \times 10^{4}$ cells were plated into the top chamber of cell culture inserts (BD Biosciences, San Diego, CA, USA) with serum-free medium. For the invasion assays, $2 \times 10^{4}$ cells were plated into the top chamber of the insert, which was precoated with Matrigel (BD Biosciences). The bottom chamber contained a $10 \%$ FBS medium. Following 2 days of incubation $5 \% \mathrm{CO}_{2}$ and $37^{\circ} \mathrm{C}$ conditions, cells adhering to the lower membrane were fixed and stained with $0.1 \%$ crystal violet. Images of the cells were captured, and the cells were counted using a light microscope (Olympus Corporation, Tokyo, Japan).

Target gene identification. TargetScan version 7.1 (www.targetscan.org/vert_71/) was used to predict the potential binding target of miRNA and genes.

Luciferase activity assays. The 3'-UTR of GRN were cloned into the PGL3 basic vector (Promega Corp., Madison, WI, USA). For the luciferase assays, $200 \mathrm{ng}$ of the PGL3-GRN-3'UTR vector
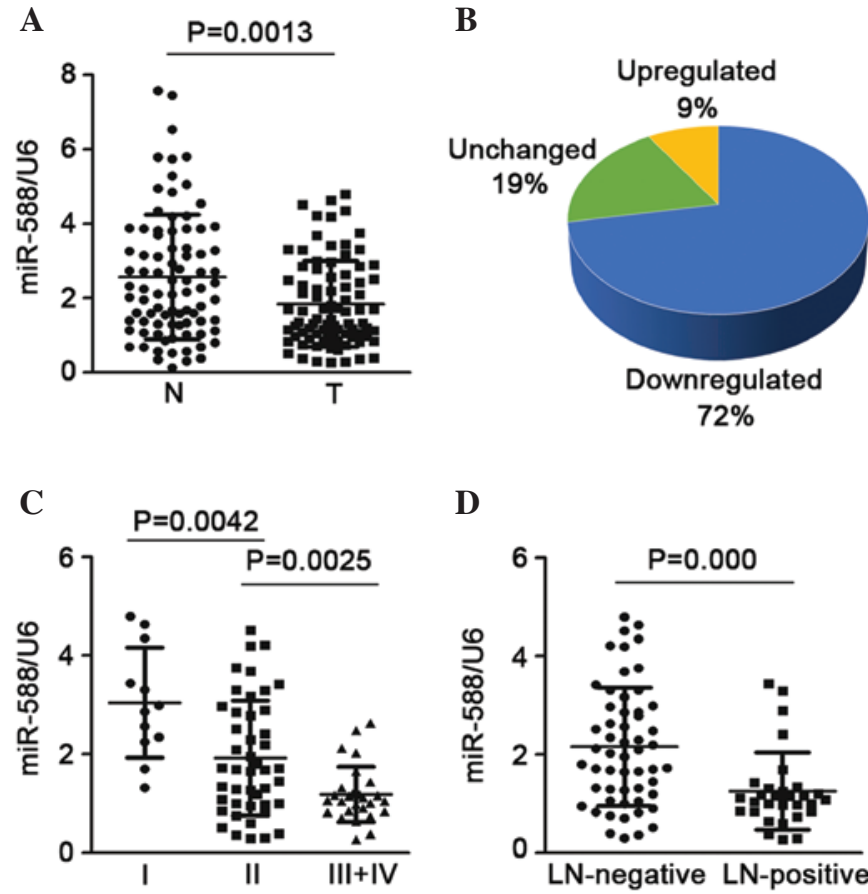

D

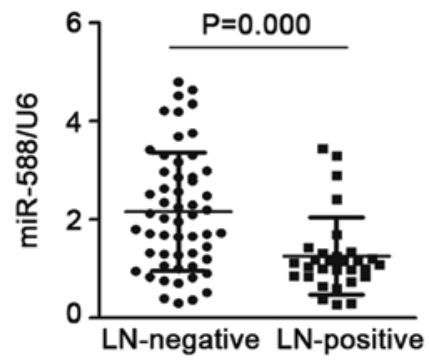

Figure 1. Expression of miR-588 is downregulated in SCC tissues and its expression is correlated with the malignant phenotype. (A) Relative mRNA levels of miR-588 in 85 paired SCC tissues and adjacent non-tumorous tissues were assessed using reverse transcription-quantitative polymerase chain reaction analysis. (B) Decreased expression of miR-588 was found in $72 \%$ (61 of 85 cases) of the SCC tissues, compared with the corresponding non-tumorous tissues. (C) Expression of miR-588 at different clinical stages of SCC. (D) Downregulation of the expression of miR-588 was associated with lymph node invasion. Data are expressed as the mean \pm standard error of the mean. miR, microRNA; SCC, squamous cell carcinoma; LN, lymph node; T, tumor; $\mathrm{N}$, non-tumor.

were co-transfected into $5 \times 10^{4}$ cells with $100 \mathrm{nM}$ miR-588 mimics or control, together with 40 ng Renilla luciferase vector (Promega Corp,) as an internal control. The cells were harvested after $48 \mathrm{~h}$ of incubation at $5 \% \mathrm{CO}_{2}$ and $37^{\circ} \mathrm{C}$ conditions following transfection and the luciferase activities were assayed according to the manufacturer's protocol. The transfections were performed in duplicate and repeated three times.

Statistical analysis. All statistical analysis was performed using SPSS 21.0 software (IBM SPSS, Armonk, NY, USA). The association between miR-588 and GRN was determined using Spearman analysis. Other experiments were repeated three times and assessed using Student's $t$-test. Data are expressed as the mean \pm standard error of the mean. $\mathrm{P}<0.05$ was considered to indicate a statistically significant difference.

\section{Results}

miR-588 is downregulated in SCC, and is correlated with advanced clinical stage and lymph node invasion. To determine the expression of miR-588 in SCC, the mature miR-588 was detected in 85 pairs of SCC tissue samples and non-tumor samples. The results showed that the expression of miR-588 was significantly downregulated in the SCC tissues, compared with the non-tumor tissues (Fig. 1A). In $\sim 72 \%$ of the samples, expression was downregulated $>2$-fold, whereas only $9 \%$ of the 

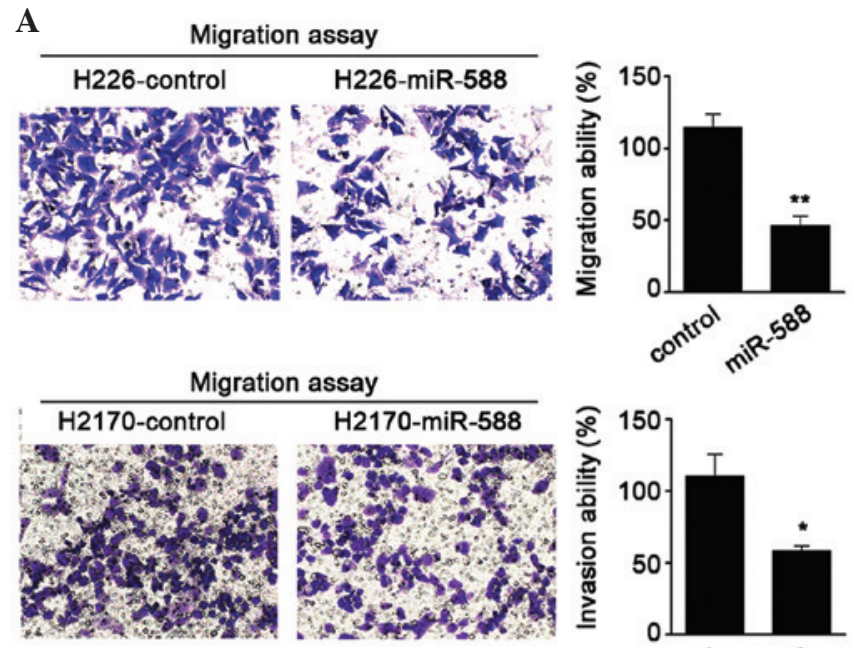

B
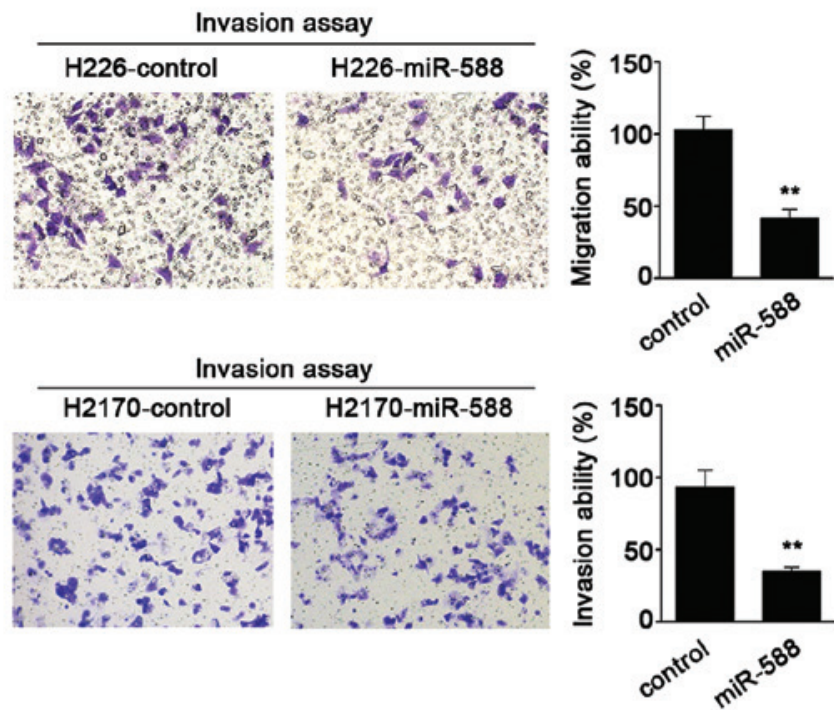

C
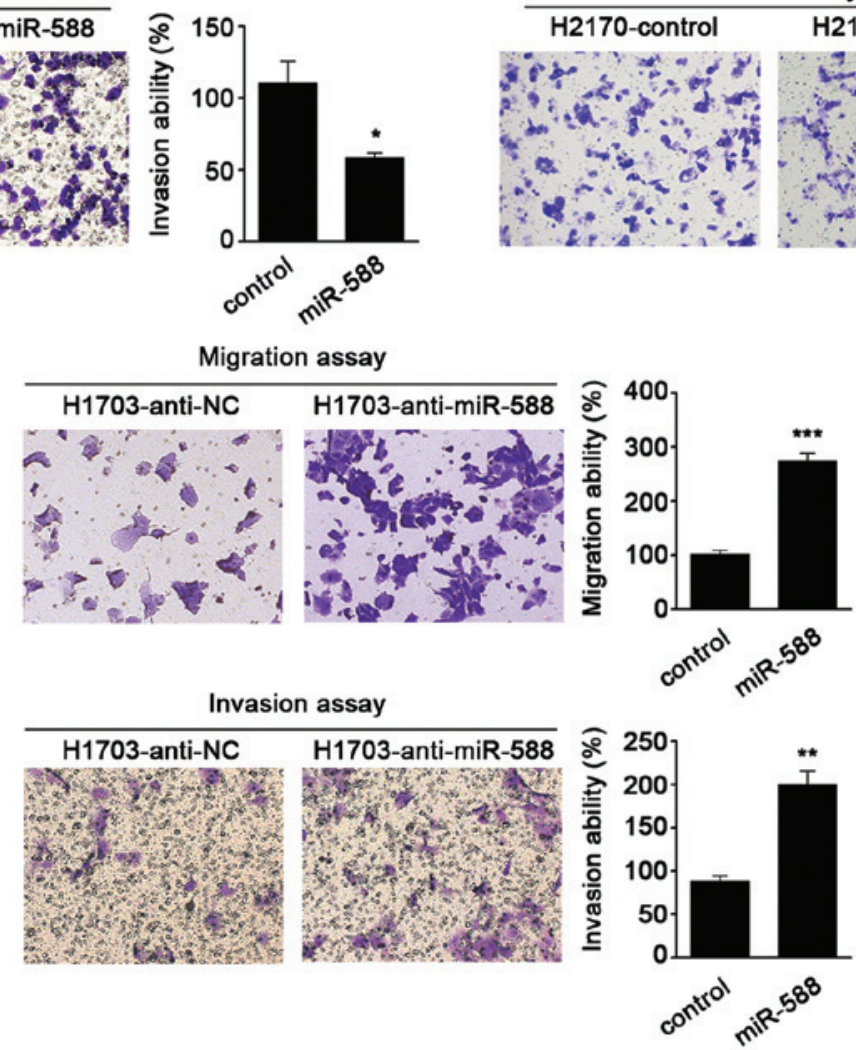

Figure 2. miR-588 suppresses SCC cell migration and invasion. Representative results of Transwell assays of the (A) migration and (B) invasion abilities of H226 and H2170 cells transfected with miR-588 mimics (magnification, x200). Results of the migration and invasion assays are shown as the average number of cells from five randomly selected fields. Data are presented as the mean \pm standard error of the mean of three independent experiments. (C) Migratory and invasive behaviors were analyzed following the inhibition of miR-588 in H1703 cells (magnification, x200). Quantification of cell migration and invasion was performed from five randomly selected fields. Each experiment was performed in triplicate and data are expressed as the mean \pm standard error of the mean $\left({ }^{*} \mathrm{P}<0.05,{ }^{* *} \mathrm{P}<0.01\right.$ and $\left.{ }^{* * *} \mathrm{P}<0.001\right)$. miR, microRNA; SCC, squamous cell carcinoma; NC, negative control.

samples showed upregulated expression (Fig. 1B). The present study also assessed the association between the expression of miR-588 and advanced stage or lymph node invasion states. It was found that SCC at an advanced stage (stages III and IV) had a lower expression level of miR-588, compared with SCC at an early stage (stage I or II; Fig. 1C). In addition, when the 85 SCC samples were cataloged based on their status of lymph node invasion, it was found that the expression of miR-588 was notably downregulated in the SCC samples, which were positive for lymph node invasion, compared with those without (Fig. 1D). Taken together, these finding suggested that the downregulation of miR-588 may be critical in the progression and metastasis of SCC.

miR-588 suppresses the migration and invasion of SCC cells. To further investigate the biological functions of miR-588 in SCC, gain- and loss-of-function analyses were performed in
SCC cells. miR-588 mimics were transfected into H226 and $\mathrm{H} 2170$ cells, as these two cells had lower relative basal expression levels of miR-588. It was found that miR-588 significantly suppressed the migration and invasion abilities of the cells (Fig. 2A and B). Antagonists were then used to inhibit the expression of miR-588 in H1703 cell lines. The results were confirmed the hypothesis that the knockdown of miR-588 increases SCC cell migration and invasion (Fig. 2C). These data suggested that miR-588 conferred negative effects on SCC metastasis.

GRN is a downstream target of miR-588 in SCC. To further examine the molecular targets of miR-588 in SCC metastasis, the TargetScan algorithm was used to predict the potential downstream gene of miR-588. GRN, a growth factor which contributes to tumor invasion and metastasis, was one of the candidates identified (Fig. 3A). RT-qPCR was then performed 
A

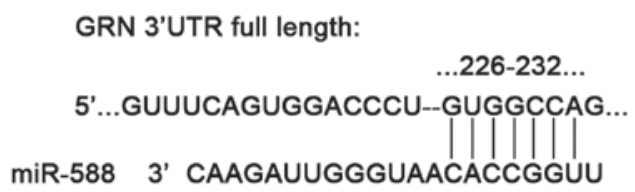

C

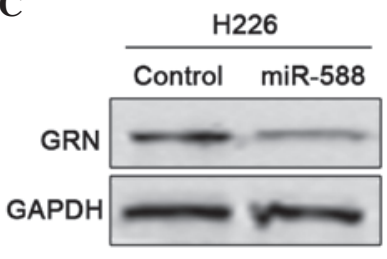

$\mathbf{E}$

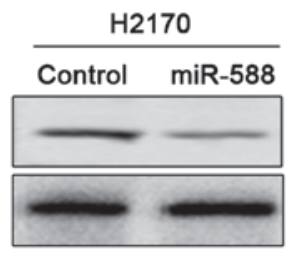

B

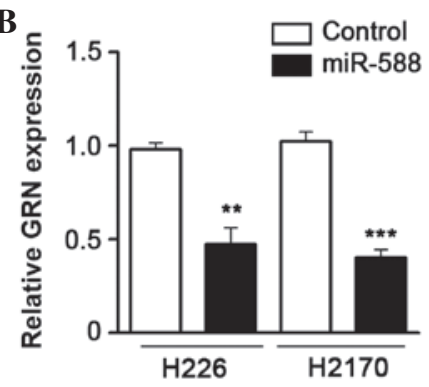

D

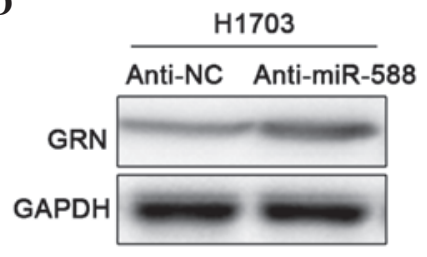

H226

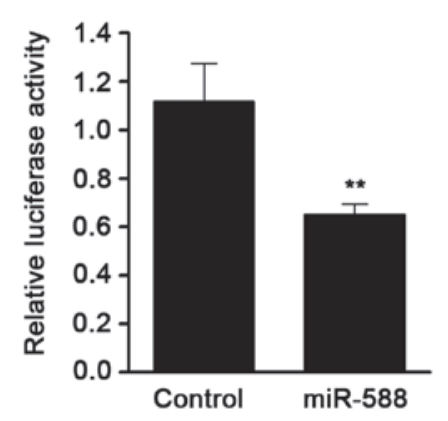

F

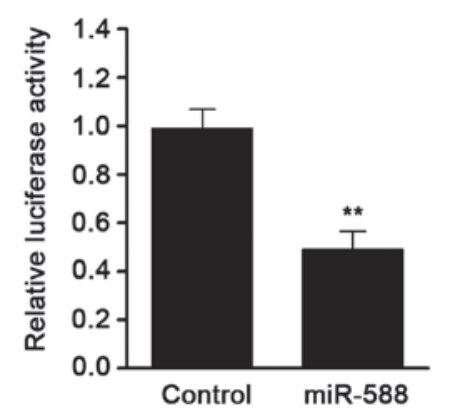

Figure 3. miR-588 negatively regulates the expression of GRN by targeting the 3'UTR of GRN. (A) Putative miR-588 binding site in the GRN 3'UTR. (B) mRNA levels of GRN were determined using reverse transcription-quantitative polymerase chain reaction analysis in miR-588 transfected H226 and $\mathrm{H} 2170$ cell lines. $\beta$-actin served as an internal control. Each experiment was performed in triplicate and values are expressed as the mean \pm standard error of the mean. (C) Western blot analysis was performed to assess the expression levels of GRN in H226 and H2170 cells transfected with control or miR-588 mimics. GAPDH served as an internal control. (D) Western blot analysis was used to detect the expression level of endogenous GRN in H1703 cells following transfection with NC inhibitor or miR-588 inhibitor. GAPDH served as an internal control. Relative luciferase activity assays of luciferase reporters with the GRN 3'UTR were performed following co-transfection with miR-588 mimics or control in (E) H226 and (F) H2170 cells. The Renilla luciferase vector was co-transfected as an internal control. Each experiment was performed in triplicate and values are expressed as the mean \pm standard error of the mean $\left({ }^{* * *} \mathrm{P}<0.01\right.$ and $\left.{ }^{* * *} \mathrm{P}<0.001\right)$. miR, microRNA; SCC, squamous cell carcinoma; GRN, progranulin; 3'UTR, 3'untranslated region; NC, negative control;

to confirm the expression of GRN when miR-588 was overexpressed by mimics. The results showed that the mRNA expression of GRN was downregulated by miR-588 (Fig. 3B). In addition, western blot assays revealed that the protein level of GRN was also reduced (Fig. 3C). The present study subsequently used an antagonist of miR-588 and found that suppressing miR-588 significantly enhanced the expression of GRN in SCC cell lines (Fig. 3D). To confirm that GRN was a direct target of miR-588, a luciferase activity assay was performed. A luciferase reporter vector containing the 3'UTR of GRN was cloned and cotransfected with miR-588 mimics into the cells. The relative luciferase activity was markedly reduced when the cells were transfected with miR-588, compared with the control (Fig. 3E and F). These data suggested that the expression of GRN was regulated by miR-588 in SCC.

Upregulation of GRN is frequent and inversely associated with the expression of miR-588 in SCC. To determine the association between the expression of GRN and miR-588 and the significance of the downregulation of miR-588 in SCC, the present study first examined the expression of GRN in six pairs of SCC and non-tumor tissues using western blot analysis. The results showed that GRN was frequently overexpressed in the SCC tissues (Fig. 4A). The sample number was then increased and immunohistochemical staining was performed to determine the protein expression of GRN in 85 pairs of SCC tissues and adjacent non-tumor tissues. Using $\mathrm{H}$ scoring statistics, it was found that the expression of GRN was significantly higher in the SCC tissues, compared with the non-tumor tissues (Fig. 4B). The representative results are shown in Fig. 4C. The correlation between the expression of miR-588 and GRN in these samples were then analyzed. Using Spearman analysis, it was found that the downregulation of GRN was correlated with the upregulatied expression of miR-588 (Fig. 4D). Taken together, these data suggested that the upregulation of GRN may be due to the repression of miR-588 in SCC.

GRN is involved in the miR-588-mediated suppression of SCC cell metastasis. It has been reported that GRN is critical in 
A

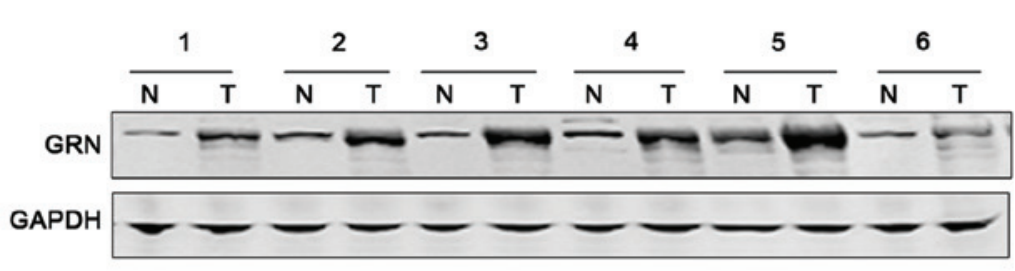

C

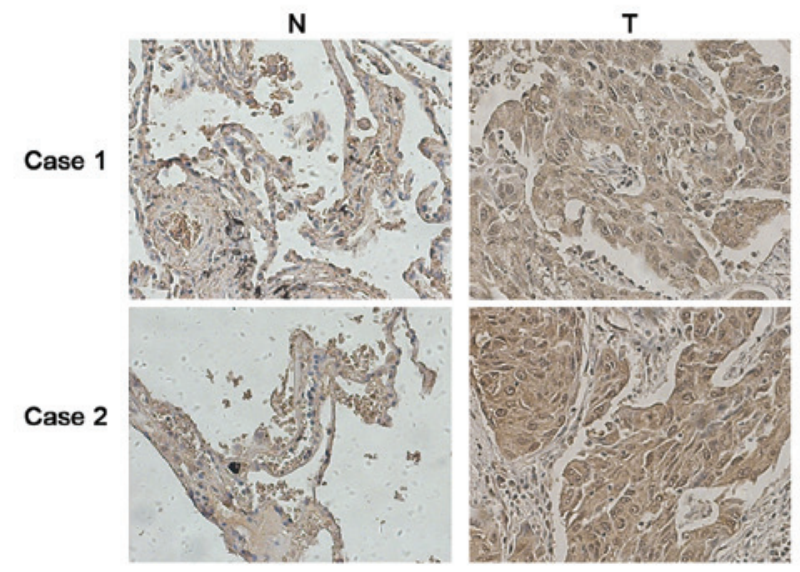

B

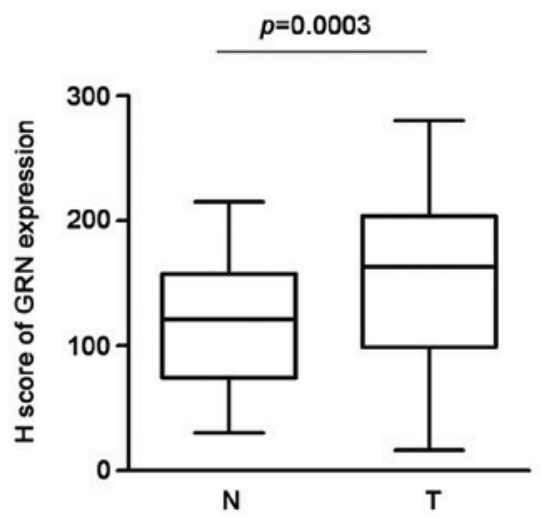

D

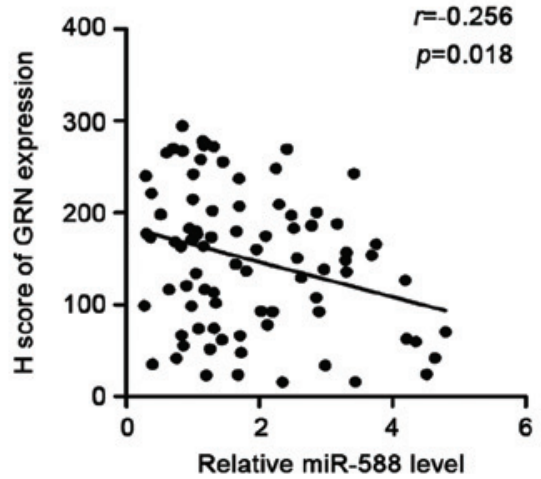

Figure 4. GRN is overexpressed in SCC tissues and inversely correlated with the expression of miR-588. (A) Representative results of western blot analysis of the expression of GRN in SCC tissues. GAPDH served as an internal control. (B) Relative scores of immunohistochemical staining of GRN in SCC tissues and adjacent non-tumor tissues. Data are expressed as the mean \pm standard error of the mean. (C) Representative images of immunohistochemical staining of GRN in SCC and adjacent non-tumorous tissues (magnification, x100). (D) Association between miR-588 and GRN, and its statistical significance were determined using Spearman analysis. miR, microRNA; SCC, squamous cell carcinoma; GRN, progranulin; 3'UTR, 3'untranslated region; N, non-tumor; T, tumor.

A

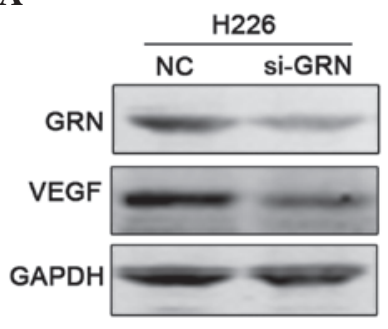

B

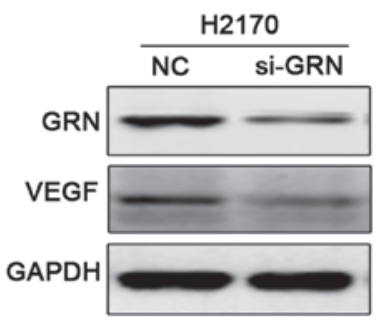

H2170

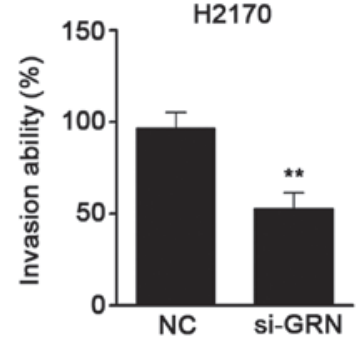

C

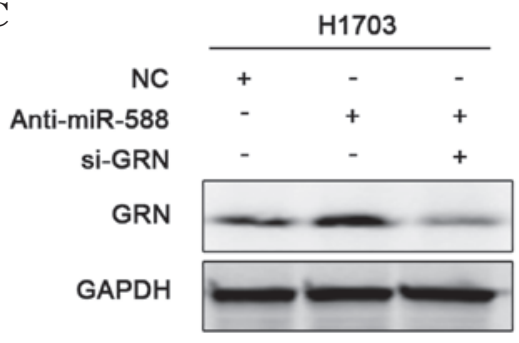

B
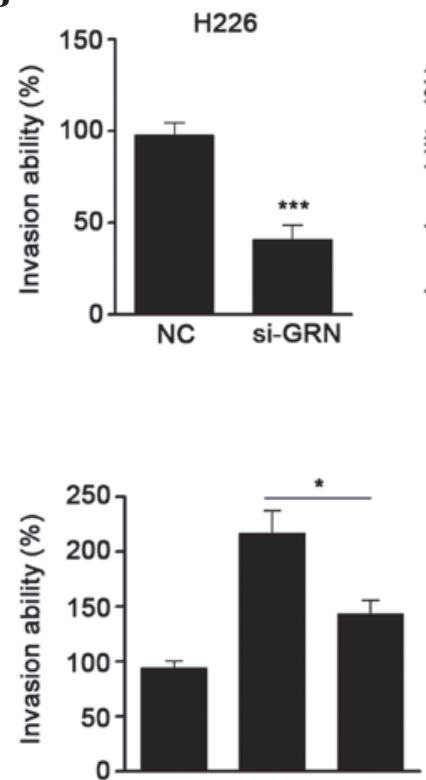

Figure 5. GRN is involved in miR-588-mediated suppression of SCC cell migration and invasion. (A) Western blot analysis was used to detect the expression levels of GRN and VEGF in H226 and H2170 cells following knockdown of GRN. GAPDH served as an internal control. (B) Invasive cells were assessed following knockdown of GRN. Results indicate the average number of invasive cells from five randomly selected fields. Data are presented as the mean \pm standard error of the mean of three independent experiments. (C) Western blot analysis was used to detect the expression of GRN following transfection with miR-588 inhibitor, si-GRN or NC in H1703 cells. GAPDH served as an internal control. Results are expressed as the average number of invasive cells from five randomly selected fields, and presented as the mean \pm standard error of the mean of three independent experiments $\left({ }^{*} \mathrm{P}<0.05,{ }^{* *} \mathrm{P}<0.01\right.$ and $\left.{ }^{* * * *} \mathrm{P}<0.001\right)$. miR, microRNA; SCC, squamous cell carcinoma; GRN, progranulin; VEGF, vascular endothelial growth factor; si-, small interfering; NC, negative control. 
stimulating cell migration and promoting metalloproteinase activity. In addition, GRN can upregulatet the expression of VEGF (24). As miR-588 was found to be downregulated in SCC, and miR-588 downregulated the expression of GRN, the present study examine whether the GRN was involved in the miR-588-mediated suppression of SCC cell metastasis. First, siRNA against GRN was used, and it was found that si-GRN significantly reduced the protein expression of GRN and the expression of VEGF. Invasion assays showed that si-GRN suppressed cell metastasis, which was similar to the results obtained when miR-588 was overexpressed (Fig. 5A and B). Subsequently, anti-miR-588 and si-GRN were transfected into H1703 cells, and the protein levels were confirmed, as shown in Fig. 5C. It was found that the enhanced metastatic ability mediated by anti-miR-588 was attenuated by si-GRN. Collectively, these results suggested that GRN was involved in the miR-588-mediated suppression of SCC cell metastasis.

\section{Discussion}

In the present study, it was shown that miR-588 was significantly decreased in SCC, particularly at advanced stages and in cases with lymph node invasion. The reinforcement of miR-588 inhibited the migration and invasion abilities of the SCC cells, and an antagonist of miR-588 promoted cell migration and invasion. Further investigations revealed that GRN was a direct target of miR-588. The expression of GRN was significantly higher in the SCC tissues, compared with the adjacent non-tumorous tissues, and was negatively correlated with the expression of miR-588. These data suggested that miR-588 acts as important negative regulator in the development and progression of SCC.

Metastasis is characterized as an important life-threatening factor in the majority of types of cancer. Lymph node invasion and angiogenesis are the major routes for SCC tumor cell metastasis $(25,26)$. Therefore, it is important to elucidate the underlying molecular mechanisms. Several studies have reported that miRNAs are associated with lymph node invasion and angiogenesis by targeting certain crucial factors. For example, miR-15a and miR-16 can target VEGF, which subsequently affects the angiogenesis of multiple myeloma (27). miR-194 downregulates the expression of matrix metalloproteinase 2/9 through targeting the expression of bone morphogenetic protein 1 and reducing the activity of transforming growth factor- $\beta$ in NSCLC (28). The present study revealed that miR-588 was complementary to the GRN 3'UTR. The in vitro restoration of miR-588 significantly suppressed cell migration and invasion. Thus, miR-588 may have potential as a treatment target for SCC.

The biological functions on miR-588 remain to be fully elucidated. Previous studies have shown that miR-588 was downregulated in cells with high metastatic capacity. Li et al (29) used an miRNA assay to scan miRNAs, which were differentially expressed between cells with high and low metastatic capacities. Their investigation found that the expression levels of miR-339-5p and miR-588 were decreased in cells with high metastatic capacity. Almog et al (30) demonstrated that high expression levels of miR-588 were shown in dormant tumors, compared with the fast-growing glioblastoma. In the presents study, miR-588 was inversely correlated with advanced tumor stage and these results were consistent with previous studies (31), indicating tumor suppression functions.
GRN has been found to be a target of miR-659 and contains common genetic variability in its miRNA binding site (32). miR-659 has been demonstrated to bind to position 83-89 of the GRN 3'UTR in complex neurodegenerative disorders, whereas miR-588 binds to position 226-232. GRN has also been reported to be regulated by miR-29b in frontotemporal dementia (33). These results suggest that multiple miRNAs may contribute to modulation of the expression of GRN. GRN has been shown be critical in pathogenesis, and as an autocrine growth and survival factor in several types of cancer (15). GRN is expressed in $70 \%$ of lung adenocarcinoma and squamous cell carcinoma tissues, whereas it is negative in normal lung tissues and in small cell carcinoma tissues (14). In addition, GRN can stimulate migration, invasiveness and the expression of VEGF in breast cancer (34). Therefore, the present study assessed the expression of VEGF when siRNA was used to knockdown GRN in SCC cells. The results provided support for the association between GRN and VEGF. It it also well documented that the expression of GRN has been associated with drug resistance in NSCLC (35). Further investigations are necessary to determine the functions of miR-588 in patients who may benefit from chemical treatment.

In conclusion, the results of the present study showed that the expression of miR-588 was downregulated in tumor tissues, compared with normal tissues, and was associated with lymph node metastasis in SCC. Furthermore, the enforced expression of miR-588 suppressed SCC cell invasion and migration through the direct targeting of GRN. These findings indicated that the downregulation of miR-588 in SCC contributed to SCC metastasis and progression, suggesting that miR-588 may be useful as a biomarker and potential therapeutic target in SCC.

\section{Acknowledgements}

This study was supported by the Scientific and Technological Innovation Programs of Higher Education Institutions in Shanxi (grant no. 20110013).

\section{References}

1. Bueno R, Hughes E, Wagner S, Gutin AS, Lanchbury JS, Zheng Y, Archer MA, Gustafson C, Jones JT, Rushton K, et al: Validation of a molecular and pathological model for five-year mortality risk in patients with early stage lung adenocarcinoma. J Thorac Oncol 10: 67-73, 2015.

2. DeSantis CE, Lin CC, Mariotto AB, Siegel RL, Stein KD, Kramer JL, Alteri R, Robbins AS and Jemal A: Cancer treatment and survivorship statistics, 2014. CA Cancer J Clin 64: 252-271, 2014.

3. Fan XX, Wong MP, Cao ZW, Li N, Wu JL, Zhou H, Jiang XH, Liu L and Leung ELH: Apoptotic effect of a single compound derived from natural product in Gefitinib-resistant non-small cell lung cancer cells. In: Proceedings of the 105th Annual Meeting of the American Association for Cancer Research, San Diego, CA, USA, 74: 3193, 2014.

4. Kim HR, Kim DJ, Kang DR, Lee JG, Lim SM, Lee CY, Rha SY, Bae MK, Lee YJ, Kim SH, et al: Fibroblast growth factor receptor 1 gene amplification is associated with poor survival and cigarette smoking dosage in patients with resected squamous cell lung cancer. J Clin Oncol 31: 731-737, 2013.

5. Tsai JH, Donaher JL, Murphy DA, Chau S and Yang J: Spatiotemporal regulation of epithelial-mesenchymal transition is essential for squamous cell carcinoma metastasis. Cancer Cell 22: 725-736, 2012. 
6. Wood SL, Pernemalm M, Crosbie PA and Whetton AD: The role of the tumor-microenvironment in lung cancer-metastasis and its relationship to potential therapeutic targets. Cancer Treat Rev 40: 558-566, 2014

7. Yang L, Li G, Zhao L, Pan F, Qiang J and Han S: Blocking the PI3K pathway enhances the efficacy of ALK-targeted therapy in EML4-ALK-positive nonsmall-cell lung cancer. Tumor Biol 35: 9759-9767, 2014

8. Gainor JF, Varghese AM, Ou SH, Kabraji S, Awad MM, Katayama R, Pawlak A, Mino-Kenudson M, Yeap BY, Riely GJ, et al: ALK rearrangements are mutually exclusive with mutations in EGFR or KRAS: An analysis of 1,683 patients with non-small cell lung cancer. Clin Cancer Res 19: 4273-4281, 2013.

9. Lu J, Getz G, Miska EA, Alvarez-Saavedra E, Lamb J, Peck D, Sweet-Cordero A, Ebert BL, Mak RH, Ferrando AA, et al: MicroRNA expression profiles classify human cancers. Nature 435: 834-838, 2005.

10. Boeri M, Verri C, Conte D, Roz L, Modena P, Facchinetti F, Calabrò E, Croce CM, Pastorino U and Sozzi G: MicroRNA signatures in tissues and plasma predict development and prognosis of computed tomography detected lung cancer. Proc Natl Acad Sci USA 108: 3713-3718, 2011.

11. Song SJ, Poliseno L, Song MS, Ala U, Webster K, Ng C, Beringer G, Brikbak NJ, Yuan X, Cantley LC, et al MicroRNA-antagonism regulates breast cancer stemness and metastasis via TET-family-dependent chromatin remodeling. Cell 154: 311-324, 2013

12. Valeri N, Braconi C, Gasparini P, Murgia C, Lampis A, Paulus-Hock V, Hart JR, Ueno L, Grivennikov SI, Lovat F, et al: MicroRNA-135b promotes cancer progression by acting as a downstream effector of oncogenic pathways in colon cancer. Cancer Cell 25: 469-483, 2014.

13. Kohlhapp FJ, Mitra AK, Lengyel E and Peter ME: MicroRNAs as mediators and communicators between cancer cells and the tumor microenvironment. Oncogene 34: 5857-5868, 2015.

14. Edelman MJ, Feliciano J, Yue B, Bejarano P, Ioffe O, Reisman D, Hawkins D, Gai Q, Hicks D and Serrero G: GP88 (progranulin): A novel tissue and circulating biomarker for non-small cell lung carcinoma. Hum Pathol 45: 1893-1899, 2014.

15. Wang M, Li G, Yin J, Lin T and Zhang J: Progranulin overexpression predicts overall survival in patients with glioblastoma. Med Oncol 29: 2423-2431, 2012.

16. Serrero G, Hawkins DM, Yue B, Ioffe O, Bejarano P, Phillips JT, Head JF, Elliott RL, Tkaczuk KR, Godwin AK, et al: Progranulin (GP88) tumor tissue expression is associated with increased risk of recurrence in breast cancer patients diagnosed with estrogen receptor positive invasive ductal carcinoma. Breast Cancer Res 14: R26, 2012.

17. Abrhale T, Brodie A, Sabnis G, Macedo L, Tian C, Yue B and Serrero G: GP88 (PC-cell derived growth factor, progranulin) stimulates proliferation and confers letrozole resistance to aromatase overexpressing breast cancer cells. BMC Cancer 11: 231, 2011.

18. Demorrow S: Progranulin: A novel regulator of gastrointestinal cancer progression. Transl Gastrointest Cancer 2: 145-151, 2013.

19. Brambilla E, Travis WD, Colby TV, Corrin B and Shimosato Y: The new World Health Organization classification of lung tumours. Eur Respir J 18: 1059-1068, 2001.

20. Goldstraw P, Crowley J, Chansky K, Giroux DJ, Groome PA, Rami-Porta R, Postmus PE, Rusch V, Sobin L; International Association for the Study of Lung Cancer International Staging Committee; Participating Institutions: The IASLC Lung Cancer Staging Project: Proposals for the revision of the TNM stage groupings in the forthcoming (seventh) edition of the TNM Classification of malignant tumours. J Thorac Oncol 2: 706-714, 2007.
21. Sartorius CA, Hanna CT, Gril B, Cruz H, Serkova NJ, Huber KM, Kabos P, Schedin TB, Borges VF, Steeg PS and Cittelly DM: Estrogen promotes the brain metastatic colonization of triple negative breast cancer cells via an astrocyte-mediated paracrine mechanism. Oncogene 35: 2881-2892, 2016.

22. Chen Q, Sun W, Liao Y, Zeng H, Shan L, Yin F, Wang Z, Zhou Z, Hua Y and Cai Z: Monocyte chemotactic protein-1 promotes the proliferation and invasion of osteosarcoma cells and upregulates the expression of AKT. Mol Med Rep 12: 219-225, 2015.

23. Jiang J, Yu C, Chen M, Tian S and Sun C: Over-expression of TRIM37 promotes cell migration and metastasis in hepatocellular carcinoma by activating Wnt/beta-catenin signaling. Biochem Biophys Res Commun 464: 1120-1127, 2015.

24. Tangkeangsirisin W and Serrero G: GP88 (progranulin) confers fulvestrant (Faslodex, ICI 182,780) resistance to human breast cancer cells. Adv Breast Cancer Res 3, 68-78, 2014.

25. Liu XG, Zhu WY, Huang YY, Ma LN, Zhou SQ, Wang YK, Zeng F, Zhou JH and Zhang YK: High expression of serum miR-21 and tumor miR-200c associated with poor prognosis in patients with lung cancer. Med Oncol 29: 618-626, 2012.

26. Zhang BC, Gao J, Wang J, Rao ZG, Wang BC and Gao JF: Tumor-associated macrophages infiltration is associated with peritumoral lymphangiogenesis and poor prognosis in lung adenocarcinoma. Med Oncol 28: 1447-1452, 2011.

27. Yang T, Thakur A, Chen T, Yang L, Lei G, Liang Y, Zhang S, Ren $\mathrm{H}$ and Chen M: MicroRNA-15a induces cell apoptosis and inhibits metastasis by targeting BCL2L2 in non-small cell lung cancer. Tumor Biol 36: 4357-4365, 2015.

28. Wu X, Liu T, Fang O, Leach LJ, Hu X and Luo Z: miR-194 suppresses metastasis of non-small cell lung cancer through regulating expression of BMP1 and p27kip1. Oncogene 33: 1506-1514, 2014

29. Li Y, Zhao W, Bao P, Li C, Ma XQ, Li Y and Chen LA: miR-339-5p inhibits cell migration and invasion in vitro and may be associated with the tumor-node-metastasis staging and lymph node metastasis of non-small cell lung cancer. Oncol Lett 8: 719-725, 2014

30. Almog N, Ma L, Schwager C, Brinkmann BG, Beheshti A, Vajkoczy P, Folkman J, Hlatky L and Abdollahi A: Consensus micro RNAs governing the switch of dormant tumors to the fast-growing angiogenic phenotype. PLoS One 7: e44001, 2012.

31. Li Y, Zhao W, Bao P, Li C, Ma XQ, Li Y and Chen LA: miR-339-5p inhibits cell migration and invasion in vitro and may be associated with the tumor-node-metastasis staging and lymph node metastasis of non-small cell lung cancer. Oncology Lett 8: 719-725, 2014

32. Rademakers R, Eriksen JL, Baker M, Robinson T, Ahmed Z, Lincoln SJ, Finch N, Rutherford NJ, Crook RJ, Josephs KA, et al: Common variation in the miR-659 binding-site of GRN is a major risk factor for TDP43-positive frontotemporal dementia. Hum Mol Genet 17: 3631-3642, 2008.

33. Jiao J, Herl LD, Farese RV and Gao FB: MicroRNA-29b regulates the expression level of human progranulin, a secreted glycoprotein implicated in frontotemporal dementia. PLoS One 5: e10551, 2010.

34. Tangkeangsirisin W and Serrero G: PC cell-derived growth factor (PCDGF/GP88, progranulin) stimulates migration, invasiveness and VEGF expression in breast cancer cells. Carcinogenesis 25: $1587-1592,2004$

35. Edelman MJ, Feliciano J, Yue B, Bejarano P, Ioffe O, Reisman D, Hawkins D, Gai Q, Hicks D and Serrero G: GP88 (progranulin): a novel tissue and circulating biomarker for non-small cell lung carcinoma. Hum Pathol 45: 1893-1899, 2014. 